\title{
A Comparison Study on the Change in Lumbar Lordosis When Standing, Sitting on a Chair, and Sitting on the Floor in Normal Individuals
}

\author{
Jun Seok Bae, M.D., Jee-Soo Jang, M.D., Ph.D., ${ }^{1}$ Sang-Ho Lee, M.D., Ph.D., Jin Uk Kim, M.D. ${ }^{2}$ \\ Department of Neurosurgery, ${ }^{1}$ Wooridul Spine Hospital, Seoul, Korea \\ Department of Orthpedic Surgery, ${ }^{2}$ Seoul Wooridul Hospital, Seoul, Korea
}

Objective : To compare radiographic analysis on the sagittal lumbar curve when standing, sitting on a chair, and sitting on the floor.

Methods : Thirty asymptomatic volunteers without a history of spinal pathology were recruited. The study population comprised 11 women and 19 men with a mean age of 29.8 years. An independent observer assessed whole lumbar lordosis (WL) and segmental lordosis (SL) between L1 and S1 using the Cobb's angle on lateral radiographs of the lumbar spine obtained from normal individuals when standing, sitting on a chair, and sitting on the floor. WL and SL at each segment were compared for each position.

Results : WL when sitting on the floor was reduced by $72.9 \%$ than the average of that in the standing position. Of the total decrease in WL, $78 \%$ occurred between $L 4$ to S1. There were significant decreases in SL at all lumbar spinal levels, except L1-2, when sitting on the floor as compared to when standing and sitting on a chair. Changes in WL between the positions when sitting on a chair and when sitting on the floor were mostly contributed by the loss of SL at the L4-5 and L5-S1 levels.

Conclusion : When sitting on the floor, WL is relatively low; this is mostly because of decreasing lordosis at the L4-5 and L5-S1 levels. In the case of lower lumbar fusion, hyperflexion is expected at the adjacent segment when sitting on the floor. To avoid this, sitting with a lordotic lumbar curve is important. Surgeons should remember to create sufficient lordosis when performing lower lumbar fusion surgery in patients with an oriental life style.

Key Words : Lumbar lordosis · Floor-sitting $\cdot$ Chair-sitting $\cdot$ Segmental lordosis.

\section{INTRODUCTION}

A cross-legged position is that in which the lower legs are folded towards the body, crossing each other at the ankle or calf, with both ankles on the floor. This posture has various names originating from different cultural backgrounds : in English, it is referred to as Indian style; in many European languages, as Turkish style; and in Korea, as Yangban style. Sitting on the floor in a cross-legged position is common in the oriental culture but not in the Western culture. Low back pain (LBP) associated with sitting on the chair has been widely studied. Some agree that prolonged static sitting and reduced lumbar lordosis are risk factors for $\mathrm{LBP}^{4,5,8,15)}$. However, to the best of our knowledge, the effect of sitting on the floor on the sagittal lumbar curve has not been studied. It is just presumed that sitting on the floor is worse for LBP than standing or sitting on a chair. This study is designed for radiographic comparison of lumbar lordosis when standing, sitting on a chair, and sitting on the floor. The aim of the present study is to determine the clinical significance of sitting on the floor on lumbar spinal alignment.

\section{MATERIALS AND METHODS}

\section{Population}

Thirty asymptomatic volunteers among the hospital staff without a history of spinal pathology were recruited. The study population comprised 11 women and 19 men with a mean age of 29.8 (SD, 6.2) years (range, 23-52 years).

- Received : September 23, $2011 \cdot$ Revised : November 17, $2011 \cdot$ Accepted : January 25, 2012

- Address for reprints : Jee-Soo Jang, M.D., Ph.D.

- Department of Neurosurgery, Wooridul Spine Hospital, 47-4 Cheongdam-dong, Gangnam-gu, Seoul 135-100, Korea

•Tel : +82-2-513-8270, Fax : +82-2-513-8146, E-mail : spinejj@@yahoo.co.kr

- This is an Open Access article distributed under the terms of the Creative Commons Attribution Non-Commercial License (http://creativecommons.org/licenses/by-nc/3.0) which permits unrestricted non-commercial use, distribution, and reproduction in any medium, provided the original work is properly cited. 


\section{Radiographic evaluation}

Lateral radiographs of the lumbar spine were obtained when standing, sitting on a chair, and sitting on the floor (Fig. 1). For radiography when standing, each person was asked to stand erect comfortably. The individual's arms were flexed; hands, placed on the clavicle; and the knees, held in extension. For chair sitting, the height of the chair was adjusted to allow each participant's hips and knees to flex approximately to $90^{\circ}$ and for their feet to rest comfortably on the floor. For floor-sitting, each person was asked to sit crossed-legged on the floor in a comfortable position. The distance from the radiographic source to the film was maintained at $230 \mathrm{~cm}$ for exposure. An independent observer measured all radiographic assessments by using a measuring program with a built-in picture-archiving communication system (PiView; INFINITT Co. Ltd., Seoul, Korea). Segmental lumbar lordosis (SL) between L1 to S1 and whole lumbar lordosis (WL) were assessed using the Cobb's angle in each position. SL was defined as the Cobb's angle between the superior endplate line of the upper vertebra and the inferior endplate line of the lower vertebra. SL at L5-S1 was defined as the Cobb's angle between the superior endplate line of L5 and the endplate line of S1. WL was defined as the Cobb's angle between the superior endplate line of L1 and the superior endplate line of S1.

\section{Statistical analysis}

Statistical analysis was performed using SPSS 14.0K (SPSS Inc., Chicago, IL, USA). A probability value of less than 0.05 was considered significant. Analysis of variance or the Kruskal-Wallis test was used for statistical analysis to compare the variables among the 3 groups. If a significant difference was found, the Bonferroni post hoc test was used to determine which groups were significantly different.

\section{RESULTS}

Tables 1 and 2 show comparison of SL and WL for each position. There were significant decreases in SL at all lumbar spine and $-12^{\circ}$, respectively. bar lordosis between each group
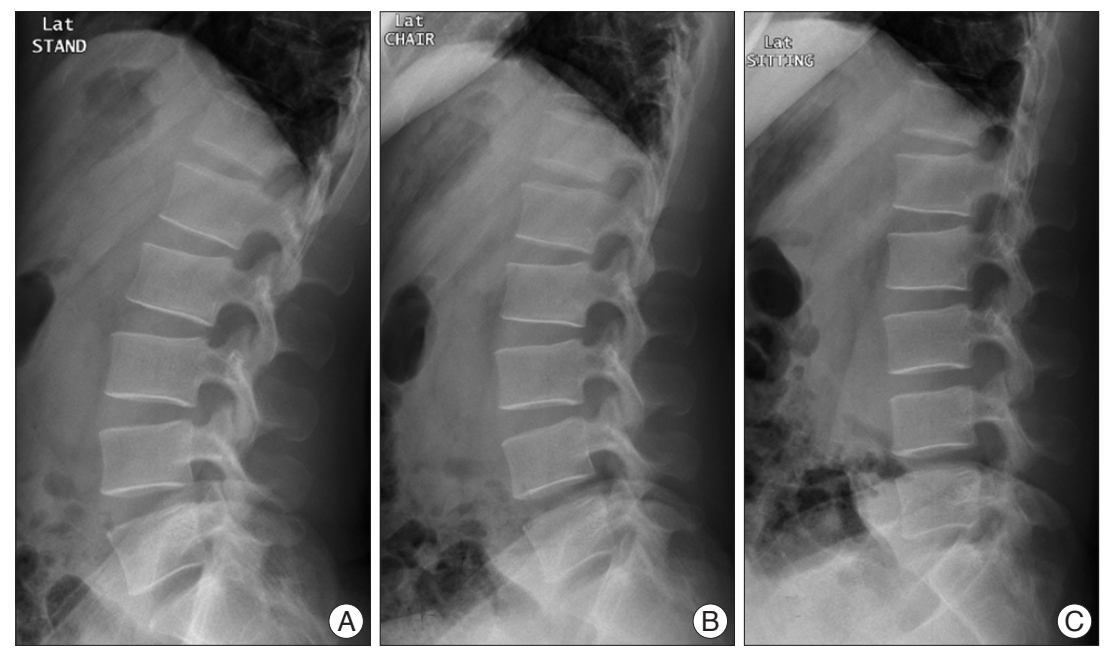

Fig. 1. A 34-year-old male participant's lateral radiographs of the lumbar spine obtained when standing (A), sitting on a chair (B), and sitting on the floor (C). In the standing position, whole lumbar lordosis (WL) is $-66^{\circ}$, and segmental lordosis (SL) at the L5-S1, L4-5, L3-4, L2-3, and L1-2 levels is $-22^{\circ},-14^{\circ},-10^{\circ},-6^{\circ}$, and $-14^{\circ}$, respectively. When sitting on a chair, WL is $-47^{\circ}$, and $S L$ at the L5$\mathrm{S} 1, \mathrm{~L} 4-5$, L3-4, L2-3, and L1-2 levels is $-9^{\circ},-5^{\circ},-9^{\circ},-7^{\circ}$, and $-17^{\circ}$, respectively. When sitting on the floor, $\mathrm{WL}$ is $-23^{\circ}$, and SL at the L5-S1, L4-5, L3-4, L2-3, and L1-2 levels is $-6^{\circ},-3^{\circ},-1^{\circ},-1^{\circ}$,

Table 1. Comparison of WL and SL among standing, chair-sitting, and floor-sitting position

\begin{tabular}{clccc}
\hline & $\begin{array}{c}\text { Standing } \\
(\mathrm{n}=30)\end{array}$ & $\begin{array}{c}\text { Chair } \\
(\mathrm{n}=30)\end{array}$ & $\begin{array}{c}\text { Floor } \\
(\mathrm{n}=30)\end{array}$ & $p$-value \\
\hline WL & $-50.0 \pm 9.2$ & $-30.2 \pm 16.0$ & $-13.9 \pm 13.6$ & $<0.0001$ \\
& $(-68.0--35.0)$ & $(-58.0-0.0)$ & $(-37.0-15.0)$ & \\
L5-S1 & $-20.2 \pm 4.5$ & $-10.3 \pm 4.3$ & $-7.4 \pm 4.8$ & $<0.0001$ \\
& $(-33.0--10.0)$ & $(-19.0--2.0)$ & $(-20.0-2.0)$ & \\
L4-5 & $-13.4 \pm 4.1$ & $-6.1 \pm 4.6$ & $-0.6 \pm 3.1$ & $<0.0001$ \\
& $(-23.0--6.0)$ & $(-14.0-4.0)$ & $(-8.0-6.0)$ & \\
L3-4 & $-8.0 \pm 2.8$ & $-5.0 \pm 3.9$ & $-1.1 \pm 3.3$ & $<0.0001$ \\
& $(-13.0--3.0)$ & $(-14.0-3.0)$ & $(-9.0-6.0)$ & \\
L2-3 & $-3.9 \pm 2.0$ & $-2.4 \pm 3.5$ & $-0.2 \pm 4.1$ & $<0.0001$ \\
& $(-10.0--1.0)$ & $(-8.0-6.0)$ & $(-9.0-9.0)$ & \\
L1-2 & $-4.4 \pm 5.6$ & $-5.5 \pm 6.8$ & $-5.0 \pm 5.6$ & 0.742 \\
& $(-16.0-1.0)$ & $(-19.0-7.0)$ & $(-15.0-5.0)$ & \\
\hline
\end{tabular}

Mean value \pm standard deviation (range) (degree). $p$-value is determined by Kruskal Wallis test. WL : whole lum-

Table 2. Post hoc test (by Bonferroni correction method) used to determine significant difference

\begin{tabular}{lccccc}
\hline$p$-value Bonf & WL & L5-S1 & L4-5 & L3-4 & L2-3 \\
\hline Standing vs. Chair & 0.000 & 0.000 & 0.000 & 0.003 & 0.300 \\
Standing vs. Floor & 0.000 & 0.000 & 0.000 & 0.000 & 0.000 \\
Chair vs. Floor & 0.000 & 0.039 & 0.000 & 0.000 & 0.042 \\
\hline
\end{tabular}

$p$-value is determined by Mann-Whitney $\mathrm{U}$ test. WL : whole lumbar lordosis

levels, except at the L1-2 level, when sitting on the floor as compared to when standing or sitting on a chair. WL when sitting on the floor was decreased to -13.9 (SD, 13.6) degrees from -50.0 (SD, 9.2) degrees observed in the standing position (72.9\% reduction). Table 3 shows the contribution of SL change to WL change during a change in the position. L5-S1 and L4-5 contributed the most to the change in WL during a change in 
Table 3. Contribution of SL change to WL change during position change

\begin{tabular}{lcccccc}
\hline & L5-S1 & L4-5 & L3-4 & L2-3 & L1-2 & $p$-value \\
\hline Standing $\rightarrow$ Chair $^{*}$ & $47.0 \pm 42.2^{\S}$ & $32.6 \pm 33.4^{\S}$ & $12.9 \pm 20.5$ & $4.3 \pm 27.8$ & $3.2 \pm 56.2$ & $<0.0001$ \\
Standing $\rightarrow$ Floor $^{\dagger}$ & $40.2 \pm 25.0^{\S}$ & $37.9 \pm 18.1^{\S}$ & $19.3 \pm 9.2$ & $9.7 \pm 10.8$ & $-7.1 \pm 29.5$ & $<0.0001$ \\
Chair $\rightarrow$ Floor $^{\ddagger}$ & $6.9 \pm 73.4$ & $62.2 \pm 80.1^{\S}$ & $33.5 \pm 61.4$ & $31.1 \pm 111.9$ & $-33.9 \pm 120.7$ & $<0.0001$ \\
\hline
\end{tabular}

Mean value \pm standard deviation. $p$-value is determined by Mann-Whitney U test. ${ }^{*}$ (Chair-Standing) $\Delta S L \Delta W L \times 100$ (\%), ${ }^{\dagger}$ (Floor-Standing) $\Delta S L / \Delta W L \times 100$ (\%), ${ }^{\ddagger}($ FloorChair) $\Delta S L \Delta \mathrm{WL} \times 100(\%) .{ }^{8}$ The most contributed level during each position change (by the Bonferroni post hoc test)

position. A change in position from standing to sitting on a chair resulted in a change in WL of a mean value of $47 \%$ and $32.6 \%$ at the L5-S1 and L4-5 levels, respectively. A change in position from standing to sitting on the floor resulted in a change in WL of a mean value of $40.2 \%$ and $37.9 \%$ at the L5-S1 and L4-5 levels, respectively. A change in position from sitting on the chair to sitting on the floor resulted in a change of $62.2 \%$ in WL at the L4-5 level.

\section{DISCUSSION}

There was more loss in WL when sitting on the floor than when sitting on the chair. Each SL below the L2-3 level also significantly decreased when sitting on the floor. Most of the WL is determined by the L4-S1 level, and most of the change in WL observed during a change in position is also attributed to the L4-S1 level.

When sitting comfortably on the floor, WL below the L1-2 level became more kyphotic and a decrease in SL was prominent at the L4-5 and L5-S1 levels. This implies relaxation of the thoracic erector spinae and lumbar multifidus muscle ${ }^{14)}$. In comparison with the chair-sitting position, SL at the L4-5 level was greatly decreased than other levels during the floor-sitting position; this reveals high compressive loading at this level.

In the case of low lumbar fusion, especially at the L4-5 level, adjacent segment hypermobility is expected when sitting on the floor. Because loss of segmental motion at the L4-5 level is shown to be redistributed among multiple cranial segments, in most cases at the first cranial adjacent level ${ }^{2)}$, individuals used to sitting on the floor are more prone to adjacent segment hypermobility after low lumbar fusion. Akamaru et al. ${ }^{1}$ demonstrated that hypolordotic fusion at the L4-5 level caused the greatest amount of flexion-extension motion at the L3-4 level. Bae et al. ${ }^{3)}$ reported that postoperative hypolordotic SL is a risk factor for adjacent segment degeneration. We speculate that hypolordotic fusion might aggravate adjacent segment motion and degeneration in the sitting position. Surgeons should remember to avoid hypolordotic low lumbar fusion in individuals who are used to sitting on the floor.

Low lumbar fusion, especially in the hypolordotic state, is highly associated with hyperflexion at the adjacent segment when sitting on the floor; this brings about deactivation of the local lumbar stabilizing muscle and increases connective tissue strain ${ }^{12}$. Deactivation of the local spinal stabilizing muscle that is known to be fatigue-resistant exerts high compressive loads on the spine ${ }^{7,10)}$.
Hence, patients may feel uncomfortable and face difficulty when sitting on the floor after low lumbar fusion surgery. This is in close agreement with numerous authors ${ }^{1,9,11}$. Loss of SL is associated with an increased incidence of low back pain and increased rate of degenerative changes observed at the adjacent levels.

To decrease segmental motion at the adjacent segment after low lumbar fusion in the sitting position, it may be beneficial to fuse the lumbar spine in normal or hyperlordotic alignment ${ }^{1)}$. O'Sullivan et al. ${ }^{14)}$ suggested a lumbopelvic sitting position in which participants were instructed to rotate their pelvis anteriorly to obtain a neutral lordosis in the lumbar spine and relax their thorax. This resulted in increased coactivation of the superficial lumbar multifidus and internal oblique and is capable of providing a local stabilizing effect on the lumbopelvic region without high compressive loading. However, this position may not be easily achieved when sitting on the floor because of ischial tuberosities. When sitting, the spinal load is carried mainly by the ischial tuberosities ${ }^{15)}$, which make it difficult to rotate the pelvis anteriorly when sitting on the floor. Makhsous et al. ${ }^{13)}$ proposed that sitting on a chair with reduced ischial support and enhanced lumbar support could decrease sitting-related low back pain. It is presumed that lumbar support provided by a cushion for sitting on the floor helps reduce sitting load and enhances neutral lumbar lordosis by rotating the pelvis anteriorly.

Contribution to the loss of lordosis when sitting on a chair and on the floor was the greatest at levels L5-S1, and it showed relatively less influence on the change in lordosis when switching positions from sitting on a chair to sitting on the floor. This implies hypermobility at the L5-S1 level in any sitting position, which explains the remarkable incidence of nonunion at this level ${ }^{6}$.

Using the clinical significance of this data, we should 1) explain to patients before they undergo surgery that they would experience difficulty when sitting on the floor after fusion surgery, 2) create greater lordotic fusion for the low lumbar spine, 3) recommend patients a change in lifestyle, and 4) perform more rigid fixation for the L5-S1 fusion.

Because this study included only healthy volunteers, there exists a limitation to explain the changes of SL and WL after low lumbar fusion. We are planning to perform similar study in fusion group and expect to report this result in near future.

\section{CONCLUSION}

The results of this study suggest sitting on the floor affects the 
sagittal lumbar curve by decreasing lordosis at each level, especially at the L4-5 and L5-S1 levels. In the case of low lumbar fusion, hyperflexion is expected at the adjacent segment when sitting on the floor. In this respect, sitting on the floor can easily aggravate LBP. To avoid this, sitting with a lordotic lumbar curve is important. Surgeons should remember to create sufficient lordosis when performing lower lumbar fusion surgery in patients with an oriental life style.

\section{References}

1. Akamaru T, Kawahara N, Tim Yoon S, Minamide A, Su Kim K, Tomita $\mathrm{K}$, et al. : Adjacent segment motion after a simulated lumbar fusion in different sagittal alignments : a biomechanical analysis. Spine (Phila Pa 1976) $28: 1560-1566,2003$

2. Auerbach JD, Jones KJ, Milby AH, Anakwenze OA, Balderston RA : Segmental contribution toward total lumbar range of motion in disc replacement and fusions : a comparison of operative and adjacent levels. Spine (Phila Pa 1976) $34:$ :2510-2517, 2009

3. Bae JS, Lee SH, Kim JS, Jung B, Choi G : Adjacent segment degeneration after lumbar interbody fusion with percutaneous pedicle screw fixation for adult low-grade isthmic spondylolisthesis : minimum 3 years of follow-up. Neurosurgery 67 : 1600-1607; discussion 1607-1608, 2010

4. Callaghan JP, McGill SM : Low back joint loading and kinematics during standing and unsupported sitting. Ergonomics $44: 280-294,2001$

5. Dunk NM, Callaghan JP : Lumbar spine movement patterns during prolonged sitting differentiate low back pain developers from matched asymptomatic controls. Work 35 : 3-14, 2010

6. Edwards CC 2nd, Bridwell KH, Patel A, Rinella AS, Berra A, Lenke LG : Long adult deformity fusions to L5 and the sacrum. A matched cohort analysis. Spine (Phila Pa 1976) 29 : 1996-2005, 2004

7. Gardner-Morse MG, Stokes IA : The effects of abdominal muscle coactivation on lumbar spine stability. Spine (Phila Pa 1976) 23 : 86-91; discussion 91-92, 1998

8. Harrison DD, Harrison SO, Croft AC, Harrison DE, Troyanovich SJ : Sitting biomechanics part I : review of the literature. J Manipulative Physiol Ther 22 : 594-609, 1999

9. Jackson RP, McManus AC : Radiographic analysis of sagittal plane alignment and balance in standing volunteers and patients with low back pain matched for age, sex, and size. A prospective controlled clinical study. Spine (Phila Pa 1976) 19: 1611-1618, 1994

10. Kavcic N, Grenier S, McGill SM : Determining the stabilizing role of individual torso muscles during rehabilitation exercises. Spine (Phila Pa 1976) $29: 1254-1265,2004$

11. Kawakami M, Tamaki T, Ando M, Yamada H, Hashizume H, Yoshida $\mathrm{M}$ : Lumbar sagittal balance influences the clinical outcome after decompression and posterolateral spinal fusion for degenerative lumbar spondylolisthesis. Spine (Phila Pa 1976) 27 : 59-64, 2002

12. Macintosh JE, Bogduk N, Pearcy MJ : The effects of flexion on the geometry and actions of the lumbar erector spinae. Spine (Phila Pa 1976) $18: 884-893,1993$

13. Makhsous M, Lin F, Bankard J, Hendrix RW, Hepler M, Press J : Biomechanical effects of sitting with adjustable ischial and lumbar support on occupational low back pain : evaluation of sitting load and back muscle activity. BMC Musculoskelet Disord 10 : 17, 2009

14. O'Sullivan P, Dankaerts W, Burnett A, Chen D, Booth R, Carlsen C, et al. : Evaluation of the flexion relaxation phenomenon of the trunk muscles in sitting. Spine (Phila Pa 1976) 31 : 2009-2016, 2006

15. Pope MH, Goh KL, Magnusson ML : Spine ergonomics. Annu Rev Biomed Eng 4 : 49-68, 2002 